\title{
Performance Analysis of A Burst-Frame-Based MAC Protocol for Ultra-Wideband Ad Hoc Networks
}

\author{
Kejie Lu, Dapeng Wu, Yuguang Fang \\ Department of Electrical and Computer Engineering \\ University Of Florida \\ Gainesville, FL 32611, USA \\ Email: \{lukejie,wu,fang\}@ece.ufl.edu.
}

\author{
Robert C. Qiu \\ Department of Electrical and Computer Engineering \\ Tennessee Technological University \\ Box 5077, Cookeville, TN 38505 \\ Email: rqiu@tntech.edu.
}

\begin{abstract}
Ultra-wideband (UWB) communication is becoming an important technology for future Wireless Personal Area Networks (WPANs). A critical challenge in high data rate UWB system design is that a receiver usually needs tens of micro-seconds or even tens of milliseconds to synchronize with transmitted signals, known as the timing acquisition problem. Such a long synchronization time will cause significant overhead, since the data rate of UWB systems is expected to be very high. To address the overhead problem, we previously proposed a general framework for MAC protocols in high data rate UWB networks. In this framework, a node can aggregate multiple upper-layer packets into a larger burst frame at the MAC layer. In this paper, we analyze the unsaturated throughput performance of a burstframe-based MAC protocol within the framework. Numerical results from the analytical method give excellent agreement with the simulation results, indicating the accuracy of our analytical method.
\end{abstract}

\section{INTRODUCTION}

In the past few years, UWB communication has received considerable attention in both academia and industry. Compared to traditional narrow band systems, UWB can provide high data rate $(>100 \mathrm{Mb} / \mathrm{s})$ with very low-power emission (less than $-41 \mathrm{dBm} / \mathrm{MHz}$ ) in a short range. These features make UWB particularly suitable for wireless personal area network (WPAN) applications. Currently, IEEE 802.15.3 working group is studying the use of UWB as an alternative physical layer technique [1].

To successfully implement a UWB system, a number of challenges must be addressed [2]. One of the critical issues is timing acquisition, which is a process of synchronizing the receiver's clock with the transmitter's clock so that the receiver can determine the boundary between two transmitted symbols. Depending on the receiver design, the acquisition time varies from tens of micro-seconds to tens of milliseconds. Evidently, for high data rate applications, the overhead of preambles will significantly reduce the efficiency of UWB networks [2].

Existing works that consider the timing synchronization issue in UWB MAC layer design include [3], [4]. In [3], the authors assumed that the UWB network can provide multiple channels through different time-hopping (TH) codes. To reduce the timing synchronization overhead, [3] proposed a link maintenance scheme in which the data channel is maintained by transmitting low-rate control packets when there is no data packet to transmit. Although the link maintenance scheme achieves good performance in the simulation, there are still some critical issues unclear in [3]. For instance, it is not clear how a node selects a TH code for its data channel in a distributed manner so as to avoid using the same TH code as that used by another node. Another potential problem is that the link maintenance scheme will increase the transmission time of the transmitter, thereby reducing the battery life and introducing extra interference. In [4], the authors studied the impact of long synchronization time on the performance of CSMA/CA and TDMA schemes used in UWB networks. However, the paper did not provide solutions to mitigate the timing acquisition problem.

To address the overhead problem, we proposed a general framework for MAC protocols in high data rate UWB networks [5]. In this framework, the MAC protocol is based on the well-known IEEE 802.11 CSMA/CA protocol. The main idea of the scheme is to assemble multiple upper-layer packets into one burst frame at the MAC layer. In contrast to the traditional approach, under which each upper-layer packet is delivered individually, transmitting multiple upper-layer packets in one frame will significantly reduce the synchronization overhead. Since performance analysis is important for MAC protocol design, this paper focuses on unsaturated performance analysis for the burst-frame-based MAC protocol, which has not been addressed previously.

Performance of CSMA/CA protocols, particularly IEEE 802.11, has been studied extensively in the literature [6]-[8]. However, until recently, most theoretical studies are focusing on saturation performance [6], [7], where a node in the network always has packets ready to be transmitted. Recently, an unsaturated analysis for IEEE 802.11 is provided in [8], where the system under study is decomposed into a queueing subsystem and a service time subsystem. In this model, the MAC layer queue is modelled as $\mathrm{M} / \mathrm{G} / 1 / \mathrm{K}$; the service time is characterized by a transfer function. However, this model is not directly applicable to the unsaturated performance analysis of our burst-frame-based MAC protocol due to the complicated queueing behavior caused by burst frames.

In this work, we take the same transfer-function approach used in [8] to analyze the unsaturated performance of our burst-frame-based MAC protocol. The major contribution of our work is that our analytical method can not only handle complicated queueing behavior but also provide accurate results, which have not been achieved previously. Our numerical results from the analytical method give good agreement with the simulation results, validating the accuracy of our analytical 
method.

The rest of the paper is organized as follows. In Section II, we briefly describe our MAC protocol for UWB networks. In Section III, we analyze the unsaturated performance of the MAC protocol. Simulation and numerical results will be shown in Section IV. Finally, Section V concludes the paper.

\section{A Burst-Frame-BASEd MAC Protocol For UWB NETWORKS}

In this section, we briefly describe a burst-frame-based MAC protocol which is a special case within the framework proposed in [5]. In this protocol, we consider only one qualityof-service (QoS) class of traffic for each destination, i.e., all packets for the same destination have the same QoS requirements. Incoming packets are first classified based on its destination, and then put into a corresponding packet queue. Suppose there are $N$ nodes in a UWB network; then we can implement $N$ packet queues in each node, among which $N-1$ queues are used for buffering packets destined to other $N-1$ nodes, and one queue is used for buffering broadcast packets. For each queue, we use tail-dropping when there is a buffer overflow.

A burst frame will be generated if the total number of packets in the queue exceeds a threshold $B_{\min }$ and the server is idle (i.e., there is no other burst waiting for transmission). In addition, we assume that the total number of packets in a burst must be smaller than or equal to a preset value $B_{\max }$. In this protocol, we require that all the packets in a burst frame have the same destination so that most existing functions of IEEE 802.11 can be re-used. To achieve the fairness among destinations, a simple round-robin scheme will be employed. When a burst assembly is finished, the burst frame will be stored in a buffer and waiting for transmission. If a burst frame is correctly received, the receiver will send one ACK frame to the transmitter.

\section{UnSATURATED PERFORMANCE ANALYSIS}

In this section, we present an analytical model for evaluating our burst-frame-based MAC protocol under different incoming traffic load. Similar to [8], we also decompose the system into a queueing subsystem and a service time subsystem. For the queueing subsystem, we extend the model for general bulk service queues [9]. Particularly, we consider that a burst can be assembled only when the total number of packets in the queueing subsystem is greater than or equal to $B_{\text {min }}$; we also consider the fact that the service time depends on the number of packets in the burst, which is not considered in [9]. For the service time subsystem, we analyze the impact of burst assembly on the probability that a server is idle, and on the service time distribution.

To facilitate our discussion, we make the following assumptions:

- There are $N$ identical nodes in the network, and any two nodes in the network can directly communicate with each other.

- At each node, packet arrivals are Poisson with rate $\lambda$ (packets/sec), and the packets have a common destination.
- The size of all packets are fixed at $\bar{P}$ (bits).

- The burst service time is an integer multiple of a preset time unit $\tau$ (sec). Here the burst service time is defined as the time interval from the time epoch that a burst is assembled to the time epoch that the burst is removed from the transmission buffer. ${ }^{1}$ A transmission buffer is located in the service time subsystem. Packets being stored in a transmission buffer means that the packets are in service.

- There is no transmission failure due to bit errors.

- The probability that a burst transmission attempt fails, denoted as $p$, does not depend on the backoff stage of the node.

- The propagation delay in UWB networks is negligible.

\section{A. The Bulk Service Queueing System}

Based on the assumptions above, we can model the queueing system in any node as an $\mathrm{M} / \mathrm{G}^{\left[B_{\min }, B_{\max }\right]} / 1 / \mathrm{K}$ queue, where $K$ is the capacity of the queue and the superscription $\left[B_{\min }, B_{\max }\right]$ means that the total number of packets in a burst is an integer in the range of $\left[B_{\min }, B_{\max }\right]$. Note that here $K$ is the total number of packets that can be stored in the queue, which does not include packets in the transmission buffer.

To analyze this queueing system, we first define the state space of the queueing system according to the status of server and the number of packets in the queue. Particularly, state $I_{k}$ means that the server is idle and there are $k$ customers waiting in the queue; while state $A_{k}$ means that the server is busy and there are $k$ customers waiting in the queue. With the packet assembly policy described in the previous section, we note that if the server is idle, then the maximum number of packets in the queue must be smaller than $B_{\min }$. Therefore, the state space is

$$
\mathbf{S}=\left\{I_{0}, I_{1}, \cdots, I_{B_{\min }-1}, A_{0}, A_{1}, \cdots, A_{K}\right\} .
$$

Let $\xi(t)(t \geq 0)$ be the state of the queueing system at time $t$; let $\delta_{n}$ be the epoch of the $n$-th burst departure. We now consider the embedded Markov process $\xi_{n}$, where $\xi_{n}$ is the state of the queueing system just before $\delta_{n}$, which is

$$
\xi_{n}=\xi\left(\delta_{n}^{-}\right) .
$$

An embedded Markov chain can then be formulated, where the state space is $\left\{A_{0}, A_{1}, \cdots, A_{K}\right\}$. Let $p_{k}^{d}(0 \leq k \leq K)$ be the steady-state probability that $\xi_{n}=A_{k}$; let $p_{i j}$ be the steady-state transition probability from state $A_{i}$ to state $A_{j}$ for all $i, j$, where $0 \leq i \leq K$ and $0 \leq j \leq K$ :

$$
p_{i j}=\lim _{n \rightarrow \infty} \operatorname{Pr}\left[\xi_{n+1}=A_{j} \mid \xi_{n}=A_{i}\right] .
$$

Consequently, $p_{k}^{d}$ can be obtained by solving the embedded Markov chain with all $p_{i j}$.

To calculate $p_{i j}$, we define $\alpha(k, b)$ as the probability that $k$ packets arrive during one burst service time, given that the

${ }^{1} \mathrm{~A}$ burst will be removed from the transmission buffer if the burst is successfully received by the destination, or if the number of burst transmission failures exceeds a pre-defined retry limit. 
burst contains $b$ packets. Let $B(t)$ be the number of packets in the first burst after $t$. Define $B_{k}=B\left(\delta_{n}\right)$ given that $\xi_{n}=k$. Here we note that $B_{k}$ depends only on the assembly policy,

$$
B_{k}= \begin{cases}B_{\min } & 0 \leq k \leq B_{\min } \\ k & B_{\min }<k \leq B_{\max } \\ B_{\max } & B_{\max } \leq k \leq K\end{cases}
$$

Since $B_{k}$ packets will be assembled into the first burst after $\delta_{n}$ if $\xi_{n}=k$, we can see that the number of packets remaining in the queue immediately after the burst is created, denoted by $K_{k}$, is

$$
K_{k}=\max \left(0, k-B_{k}\right) .
$$

Therefore, we can calculate all $p_{i j}$ through

$$
p_{i j}=\left\{\begin{array}{ll}
0 & 0 \leq j<K_{i} \\
\alpha\left(j-K_{i}, B_{i}\right) & K_{i} \leq j<K \\
1-\sum_{k=K_{i}}^{K-1} \alpha\left(k-K_{i}, B_{i}\right) & j=K
\end{array} .\right.
$$

Let $q_{b i}$ be the steady state probability that the burst service time is $i \tau$, given that there are $b$ packets in the burst. Since the packet arrival is a Poisson process with rate $\lambda, \alpha(k, b)$ can be calculated by

$$
\alpha(k, b)=\sum_{\forall i} q_{b i} \times \frac{(\lambda i \tau)^{k} e^{-\lambda i \tau}}{k !}
$$

In summary, we note that $p_{k}^{d}$ can be calculated if all $q_{b i}$ are known.

\section{B. Exponential Backoff Scheme}

Using the Markov modelling technique introduced in [6], we can analyze the exponential backoff scheme for the MAC protocol. Specifically, we can partition the continuous time axis into slots, where two consecutive slots are delimited by the event of a value change in the backoff counter. We can then formulate a two-dimensional discrete time embedded Markov chain as in [7], where the maximum number of retries for a packet is taken into consideration. By solving the Markov chain, a closed-form solution for the probability that a node will transmit a burst given that the node is busy, denoted as $p_{t}$, can be achieved if the burst transmission probability $p$ is known ${ }^{2}$.

Since a successful packet delivery means that there is no collision, we can calculate $p$ through

$$
p=1-\left[1-\left(1-p_{I}\right) p_{t}\right]^{N-1}
$$

where $p_{I}$ denotes the probability that a node is idle (no burst pending for transmission) in a slot. With $0<p<1$ and $0<p_{t}<1$, we can calculate $p$ and $p_{t}$ numerically.

To calculate $p_{I}$, we let $d_{k}(t)(0 \leq k \leq K)$ be the total number of burst departures in time $(0, t)$ such that the number of packets in the queue is $k$ just before the departure; let $D(t)=\sum_{k} d_{k}(t)$ be the total number of burst departures in $(0, t)$. Note that if there are $k\left(k<B_{\text {min }}\right)$ packets in the queue just before a departure, then the average time from the

\footnotetext{
${ }^{2}$ Please refer to [7] for detail discussion.
}

departure of the old burst to the epoch that a new burst if formed is $\left(B_{\min }-k\right) \times \frac{1}{\lambda}$, since packet arrivals are Poisson with rate $\lambda$. We can then estimate $p_{I}$ as the fraction of time that the server is idle, which is

$$
\begin{aligned}
p_{I} & =\lim _{t \rightarrow \infty}\left[\frac{1}{t} \times \sum_{k=0}^{B_{\min }-1}\left(d_{k}(t) \times\left(B_{\min }-k\right) \times \frac{1}{\lambda}\right)\right] \\
& =\frac{1}{\lambda} \times \sum_{k=0}^{B_{\min }-1}\left[\left(B_{\min }-k\right) \times \lim _{t \rightarrow \infty} \frac{d_{k}(t)}{t}\right]
\end{aligned}
$$

Similar to [9], $\lim _{t \rightarrow \infty} \frac{d_{k}(t)}{t}$ can be calculated by

$$
\lim _{t \rightarrow \infty} \frac{d_{k}(t)}{D(t)} \times \lim _{t \rightarrow \infty} \frac{D(t)}{t}=p_{k}^{d} \times\left[\lim _{t \rightarrow \infty} \frac{t}{D(t)}\right]^{-1} .
$$

Since $D(t)$ is the total number of served bursts in $t$, $\lim _{t \rightarrow \infty} \frac{t}{D(t)}$ is the average burst departure interval. Therefore, we have

$$
\lim _{t \rightarrow \infty} \frac{t}{D(t)}=T^{s}+\sum_{k=0}^{B_{\min }-1} p_{k}^{d}\left[\left(B_{\min }-k\right) \frac{1}{\lambda}\right]
$$

where $T^{s}$ denotes the average burst service time, which is

$$
T^{s}=\sum_{k=0}^{K} p_{k}^{d}\left[\sum_{\forall i} q_{B_{k} i} \times(i \tau)\right] .
$$

Finally, $p_{I}$ can be calculated through

$$
p_{I}=\frac{\sum_{k=0}^{B_{\text {min }}-1}\left(B_{\min }-k\right) p_{k}^{d}}{\lambda T^{s}+\sum_{k=0}^{B_{\min }-1}\left(B_{\min }-k\right) p_{k}^{d}} .
$$

To summarize this subsection, we note that $p$ and $p_{t}$ can be calculated if all $p_{k}^{d}$ are known.

\section{Service Time Distribution}

Let $Q_{b}(z)$ be the probability-generating function (PGF) of $q_{b i}$, which is

$$
Q_{b}(z)=\sum_{i} z^{i} \cdot q_{b i}
$$

Due to the simplicity of calculation in the z-transform domain and the one-to-one correspondence between $q_{b i}$ and $Q_{b}(z)$, we compute $Q_{b}(z)$ instead of $q_{b i}$. This approach is known as the transfer-function approach [8].

To calculate $Q_{b}(z)$, we let $X_{n}$ be the length of slot $n$ and let $X_{n}^{\prime}$ be the length of a period (within slot $n$ ), during which the server is busy. Note that for saturated condition, $X_{n} \equiv X_{n}^{\prime}$. However, for unsaturated cases, $X_{n}^{\prime} \leq X_{n}$. We can then apply the technique used in [8]. In this model, the packet transmission process is characterized by a linear system, where $H(z)$ is the PGF of $X_{n}^{\prime}$ given that the current node is active but not transmitting; $C_{b}(z)$ is the PGF of $X_{n}^{\prime}$ given that a collision occurs and the current node has transmitted a burst that has $b$ packets; and $S_{b}(z)$ is the PGF of $X_{n}^{\prime}$ given that 
the current node has successfully transmitted a burst with $b$ packets. Consequently, the transfer function of the linear system is equal to $Q_{b}(z)$.

To simplify the notation, we define $H_{m}(z)$ as follows

$$
H_{m}(z)=\frac{1}{W_{m}}\left[1+H(z)+H^{2}(z)+\cdots+H^{W_{m}-1}(z)\right] .
$$

Then $Q_{b}(z)$ can then be calculated through

$$
\begin{aligned}
Q_{b}(z)= & (1-p) S_{b}(z) \sum_{m=0}^{M}\left[\left(p C_{b}(z)\right)^{m} \prod_{i=0}^{m} H_{i}(z)\right] \\
& +\left(p C_{b}(z)\right)^{M+1} \prod_{i=0}^{M} H_{i}(z)
\end{aligned}
$$

We now consider the calculation of $S_{b}(z), C_{b}(z)$, and $H(z)$. Due to limited space, here we only discuss the calculation for the RTS/CTS access scheme. Since the packet size is fixed, $S_{b}(z)$ and $C_{b}(z)$ can be directly derived as

$$
\begin{aligned}
S_{b}(z) & =z^{\left\lfloor\left(T_{s o}+\frac{b \bar{P}}{R}\right) \frac{1}{\tau}\right\rfloor} \\
C_{b}(z) & =z^{\left\lfloor\frac{T_{c o}}{\tau}\right\rfloor}
\end{aligned}
$$

where $R$ is the data rate, $T_{s o}$ is the time overhead for a successful transmission, and $T_{c o}$ is the time overhead for collision. According to IEEE 802.11 protocol, we have

$$
\begin{aligned}
T_{\text {so }}= & 4 T_{\text {sync }}+3 T_{S I F S}+T_{D I F S} \\
& +\frac{1}{R}\left(4 L_{P H}+L_{R T S}+L_{C T S}+L_{A C K}+L_{M H}\right) \\
T_{c o}= & 2 T_{\text {sync }}+T_{S I F S}+T_{D I F S} \\
& +\frac{1}{R}\left(2 L_{P H}+L_{R T S}+L_{C T S}\right)
\end{aligned}
$$

where $T_{\text {sync }}$ denotes the synchronization time, $T_{S I F S}$ denotes the time duration of SIFS, $T_{D I F S}$ denotes the time duration of DIFS, $L_{P H}$ denotes the length of physical frame header in bits (excluding the synchronization preamble), $L_{M H}$ denotes the length of MAC frame header in bits, $L_{R T S}$ denotes the length of ACK frame in bits, $L_{C T S}$ denotes the length of ACK frame in bits, and $L_{A C K}$ denotes the length of ACK frame in bits.

To calculate $H(z)$, we define the following parameters:

- $q_{t}$ denotes the probability that there is at least one packet transmission in $N-1$ nodes in a slot, which is

$$
q_{t}=1-\left[1-\left(1-p_{I}\right) p_{t}\right]^{N-1}
$$

- $q_{s}$ denotes the probability that there is only one packet transmission in $N-1$ nodes in a slot, which is

$$
q_{s}=(N-1)\left(1-p_{I}\right) p_{t}\left[1-\left(1-p_{I}\right) p_{t}\right]^{N-2}
$$

- $\sigma$ denotes the length of a preset fixed time duration for backoff. When there is no packet transmission, we have $X_{n}=\sigma$. In $802.11 \mathrm{~b}$ direct sequence spread spectrum mode, $\sigma=20 \mu s$.

With these parameters, we have

$$
H(z)=\left(1-q_{t}\right) z^{\left\lfloor\frac{\sigma}{\tau}\right\rfloor}+q_{s} S(z)+\left(q_{t}-q_{s}\right) C(z)
$$

TABLE I

SETTING OF THE MAC PROTOCOL.

\begin{tabular}{|c|c|}
\hline Minimum contention window size & 8 \\
\hline Maximum contention window size & 256 \\
\hline$\sigma$ & $2 \mu \mathrm{s}$ \\
\hline SIFS & $1 \mu \mathrm{s}$ \\
\hline DIFS & $5 \mu \mathrm{s}$ \\
\hline Retry limit & 4 \\
\hline Queue size & 50 \\
\hline
\end{tabular}

where $C(z)=C_{b}(z)$ and

$$
S(z)=\sum_{b=B_{\min }}^{B_{\max }} p_{b} \times S_{b}(z)
$$

where $p_{b}=\sum_{\forall k: B_{k}=b} p_{k}^{d}$ is the probability mass function of the number of packets in a burst.

To summarize this subsection, we note that the service time distribution $q_{b i}$ can be calculated if $p, p_{t}$, and all $p_{k}^{d}$ are known.

\section{Throughput Analysis}

Let $S(t)$ be the total number of successfully transmitted packets in $[0, t]$. Then we have

$$
S(t)=\sum_{k=0}^{K} d_{k}(t) \times B_{k} \times\left(1-p^{M+1}\right)+B(0)-B(t) .
$$

Now define throughput $S$ as the total amount of data (in bits) of MAC payload successfully received in a given time (in sec). Based on Eqs. (20), (7), and (8), we have

$$
S=\lim _{t \rightarrow \infty} \frac{S(t)}{t} \cdot \bar{P}=\frac{\bar{P} \cdot E[B] \cdot\left(1-p^{M+1}\right)}{T^{s}+\frac{1}{\lambda} \cdot \sum_{k=0}^{B_{\min }-1}\left(B_{\min }-k\right) p_{k}^{d}}
$$

where $E[B]$ is the average number of packets in a burst $E[B]=\sum_{b=B_{\min }}^{B_{\max }}\left[b \times p_{b}\right]$.

\section{E. Summary of the Algorithm}

In this analytical model, the performance is obtained through a recursive algorithm. The algorithm can be summarized as follows

1) Initialize $p_{I}$ and $p_{b}$ to saturated condition, i.e., let $p_{I}=$ $0, p_{B_{\max }}=1$, and $p_{b}=0$ for $b \neq B_{\max }$.

2) Calculate $p$ and $p_{t}$ as shown in Section III-B.

3) Calculate service time distribution $q_{b i}$, using the transferfunction approach.

4) Calculate $p_{k}^{d}$ by using the bulk service queueing model.

5) Calculate $p_{I}$ through Eq. (10).

6) Calculate $S$ through Eq. (21) and stop if the result converges, i.e., the difference between the results of two consecutive iterations is less than a preset value; otherwise go to Step 2.

Although the convergence of the recursive algorithm has not been proved, the algorithm always achieves convergence in our numerical calculations. 


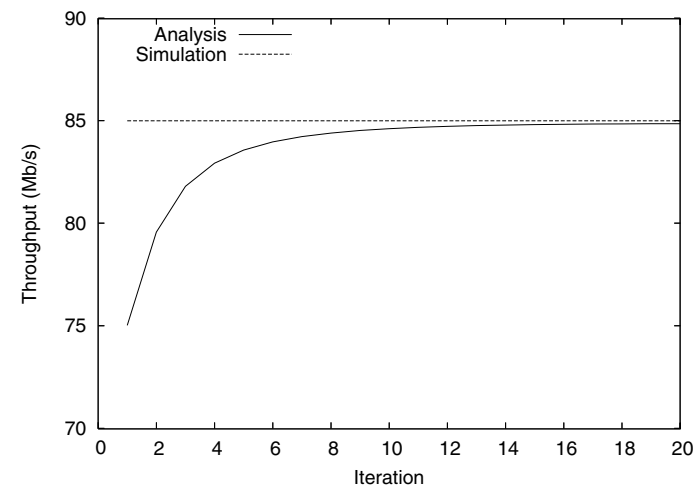

Fig. 1. Analytical results vs. number of iterations $\left(B_{\min }=1, B_{\max }=\right.$ $\left.10, R_{i}=90 \mathrm{Mb} / \mathrm{s}\right)$

\section{Simulation And Numerical Results}

In this section, we evaluate the performance of the burstframe-based MAC protocol and compare the numerical results of the proposed analytical method in Section III, to the simulation results. Table I lists the values of the control parameters used in the simulations and numerical analysis.

Due to the space limit, we only present results under the following setting:

- $N=10$ nodes are located in a $4 \mathrm{~m} \times 4 \mathrm{~m}$ area.

- All messages are transmitted with channel data rate $R$, which is $100 \mathrm{Mbits} / \mathrm{s}$.

- The size of each packet is fixed at 1000 Bytes.

- Packet arrivals to any node $i$ are modelled by a Poisson process with the same rate $\lambda$ (packets/s). Consequently, the incoming traffic data rate is $R_{i}=N \bar{P} \lambda$ (bits/s).

- We assume that $T_{\text {sync }}=10 \mu \mathrm{s}$, which is a typical assumption in [1].

- We assume that no packet transmission failure occurs due to bit errors.

- We assume that the RTS/CTS scheme is used.

For the analytical model, we let time unit $\tau=\sigma$, let the maximum service time be 30000 time units, and run the algorithm for 20 iterations.

Figure 1 shows the analytical results versus the number of iterations, where we let $B_{\min }=1, B_{\max }=10$, and $R_{i}=90$ $\mathrm{Mb} / \mathrm{s}$. We can observe that, if the number of iterations is larger than 15 , the analytical result converges, and is very close to the simulation result.

Figure 2 compares the throughput performance versus incoming traffic rate for three burst assembly policies: 1) $B_{\min }=B_{\max }=1$ (benchmark), 2) $B_{\min }=1, B_{\max }=$ 10, 3) $B_{\min }=B_{\max }=10$. We can see that, compared to the bench mark case, the proposed MAC protocol can significantly improve the throughput performance. Particularly, if $R_{i}=R$, the bench mark throughput is only about $52 \mathrm{Mb} / \mathrm{s}$; while policy (2) and (3) can achieve as high as $88 \mathrm{Mb} / \mathrm{s}$ and $92 \mathrm{Mb} / \mathrm{s}$, respectively. From Fig. 2 we can also observe that our analytical model is highly accurate under different incoming data rates. Such accuracy has not been achieved by

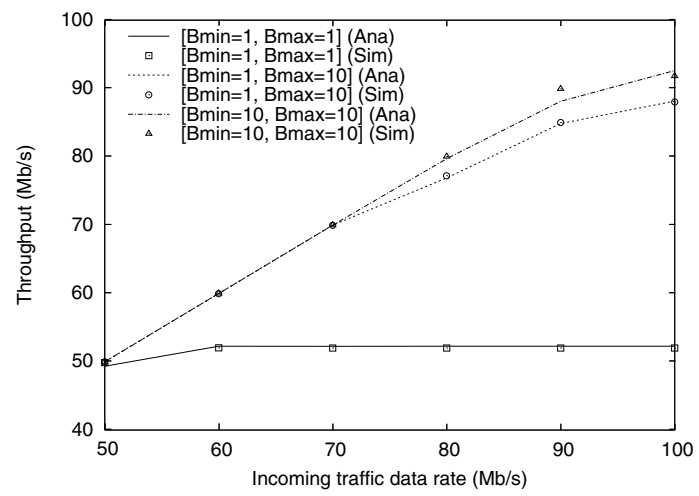

Fig. 2. Throughput performance versus incoming traffic data rate with different burst assembly policies.

the previous analysis; hence our analysis represents a major contribution.

\section{CONCLUSIONS}

In this paper, we developed an analytical model to evaluate the performance of a burst-frame-based MAC protocol, in which a node can aggregate multiple upper-layer packets into a larger burst frame at the MAC layer. The proposed theoretical model can analyze the unsaturated throughput performance of the burst-frame-based MAC protocol, which has not been addressed previously. In addition, the proposed method is general and hence applicable to unsaturated performance analysis for other CSMA/CA based protocols. Numerical results from the analytical method give excellent agreement with the simulation results, validating the accuracy of our analytical model.

\section{REFERENCES}

[1] IEEE P802.15-04/0137r1, "DS-UWB physical layer submission to 802.15 Task Group 3a,” Mar. 2004, Project: IEEE P802.15 Working Group for Wireless Personal Area Networks (WPANs).

[2] S. Roy, J. R. Foerster, V. S. Somayazulu, and D. G. Leeper, "Ultrawideband radio design: The promise of high-speed, short-range wireless connectivity," Proc. IEEE, vol. 92, no. 2, pp. 295-311, Feb. 2004.

[3] S. S. Kolenchery, J. K. Townsend, and J. A. Freebersyser, "A novel impulse radio network for tactical military wireless communications," in Proc. IEEE MILCOM'1998, 1998, pp. 59-65.

[4] J. Ding, L. Zhao, S. Medidi, and K. Sivalingam, "MAC protocols for ultra wideband (UWB) wireless networks: Impact of channel acquisition time," in Proc. SPIE ITCOM'2002, July 2002.

[5] K. Lu, D. Wu, and Y. Fang, "A novel framework for medium access control in ultra-wideband ad hoc networks," Dynamics of Continuous, Discrete and Impulsive Systems (Series B) Special Issue on Ultra-Wideband (UWB) Wireless Communications for Short Range Communications, (to appear), 2005. [Online]. Available: http://www.wu.ece.ufl.edu/mypapers/uwb_camera.pdf

[6] G. Bianchi, "Performance analysis of the ieee 802.11 distributed coordination function," IEEE J. Select. Areas Commun., vol. 18, no. 3, pp. 535-547, Mar. 2000.

[7] H. Wu, Y. Peng, K. Long, S. Cheng, and J. Ma, "Performance of reliable transport protocol over IEEE 802.11 wireless LAN: analysis and enhancement," in Proc. IEEE INFOCOM, June 2002, pp. 599-607.

[8] H. Zhai, Y. Kwon, and Y. Fang, "Performance analysis of IEEE 802.11 MAC protocol in wireless LAN," Wiley Journal of Wireless Communications and Moble Computing (WCMC), pp. 917-931, Dec. 2004.

[9] G. Hebuterne and C. Rosenberg, "Arrival and departure state disributions in the general bulk-service queue," Naval Research Logistics, vol. 46, pp. 107-118, 1999. 